\title{
On Stability of Nonautonomous Perturbed Semilinear Fractional Differential Systems of Order $\alpha \in(1,2)$
}

\author{
Mohammed M. Matar (iD) and Esmail S. Abu Skhail \\ Mathematics Department, Al-Azhar University-Gaza, State of Palestine \\ Correspondence should be addressed to Mohammed M. Matar; mohammed_mattar@hotmail.com
}

Received 13 May 2018; Accepted 25 July 2018; Published 5 August 2018

Academic Editor: Morteza Khodabin

Copyright @ 2018 Mohammed M. Matar and Esmail S. Abu Skhail. This is an open access article distributed under the Creative Commons Attribution License, which permits unrestricted use, distribution, and reproduction in any medium, provided the original work is properly cited.

\begin{abstract}
We study the Mittag-Leffler and class-K function stability of fractional differential equations with order $\alpha \in(1,2)$. We also investigate the comparison between two systems with Caputo and Riemann-Liouville derivatives. Two examples related to fractional-order Hopfield neural networks with constant external inputs and a marine protected area model are introduced to illustrate the applicability of stability results.
\end{abstract}

\section{Introduction}

Recently, the topic of fractional differential equations has attracted many researchers which plays a main role in various applications. Many areas such as physics, biophysics, aerodynamics, control theory, viscoelasticity, capacitor theory, electrical circuit, description of memory, and hereditary properties (see [1-4]) used the fractional models instead of classical models. Recently, stability of fractional differential systems has attracted many authors for more investigation in this topic [5]. In 1996, Matignon [6] firstly studied the stability of linear fractional differential systems with the Caputo derivative. Since then, many researchers have done further studies on the stability of linear fractional differential systems (see [7, 8]). In nonlinear systems, Lyapunov's direct method provides a way to analyze the stability as MittagLeffler stability of a system without explicitly solving the differential equations (see [9-11]). The method generalizes the idea which shows that the system is stable if there are some Lyapunov function candidates for the system. Lyapunov's direct method is a sufficient condition to show the stability of the systems, which means that the systems may still be stable even if one cannot find Lyapunov's function candidate to conclude the system stability property. For the nonlinear fractional differential systems, the stability analysis is much more difficult and only a few are available.
Some authors (see [12-14]) studied the following nonlinear fractional differential system:

$$
{ }^{c} D_{t_{0}, t}^{\alpha} x(t)=f(t, x(t)), \quad t \in\left[t_{0}, \infty\right) .
$$

with initial values $x^{(k)}\left(t_{0}\right)=x_{k}, k=1,2, \ldots, n-1$, where $n-1<\alpha<n$. Then, some literatures on the stability of linear fractional differential systems with $0<\alpha<1$ have appeared (see $[15,16]$ ). However, not all the fractional differential systems have fractional orders in $(0,1)$. There exist fractional models which have fractional orders lying in $(1,2)$, for example, super-diffusion. In [17], the authors study the stability of $n$-dimensional linear fractional differential systems

$$
{ }^{R L} D_{t_{0}, t}^{\alpha} x(t)=A x(t),
$$

of order $1<\alpha<2$ and initial conditions

$$
\left.{ }^{R L} D_{t_{0}, t}^{\alpha-k} x(t)\right|_{t=t_{0}}=x_{k-1}, \quad k=1,2 .
$$

The corresponding perturbed system is

$$
\begin{aligned}
{ }^{R L} D_{t_{0}, t}^{\alpha} x(t) & =A x(t)+f(t, x(t)), \quad 1<\alpha<2, \\
\left.{ }^{R L} D_{t_{0}, t}^{\alpha-k} x(t)\right|_{t=t_{0}} & =x_{k-1}, \quad k=1,2,
\end{aligned}
$$

which is investigated by authors in [17] 
For fractional-order Hopfield neural networks in applications to parallel computation and signal processing, it is required that there be a well-defined computable solution for all possible initial states. From a mathematical viewpoint, the stability analysis of a unique equilibrium point is very necessary and valuable. Recently, several important and interesting results for the stability analysis of fractional-order Hopfield neural networks have been gained [4].

The marine protected area model [18] can describe the ecological linkage between the reserve and fishing ground by the autonomous linear system

$$
\begin{aligned}
& x_{1}^{\prime}(t)=-\left(m_{1}+D_{1}\right) x_{1}(t)+D_{2} x_{2}(t), \\
& x_{2}^{\prime}(t)=-\left(m_{2}+D_{2}+E\right) x_{2}(t)+D_{1} x_{1}(t) .
\end{aligned}
$$

Here, $m_{i}>0$ is the natural mortality (death) rate, $D_{i}>0$ is the dispersal rate, and $E>0$ is the harvesting rate. The corresponding fractional behavior of such system may exist in theoretical view as the fractional order $\alpha \longrightarrow 1^{ \pm}$.

Motivated by these articles, we investigate the stability of the nonautonomous perturbed fractional semilinear system of the form

$$
\begin{aligned}
D_{t_{0}, t}^{\alpha} x(t) & =A x(t)+f(t, x(t)), \quad t>t_{0,}, \\
x\left(t_{0}\right) & =x_{0}, \\
x^{\prime}\left(t_{0}\right) & =x_{1},
\end{aligned}
$$

where $x(t) \in \mathbb{R}^{n}, x_{k} \in \mathbb{R}^{n}(k=0,1), A \in \mathbb{R}^{n \times n}, 1<\alpha<2$, and $f:\left[t_{0}, \infty\right) \times \mathbb{R}^{n} \longrightarrow \mathbb{R}^{n}$ is a given continuous function such that $f(t, 0)=0$. The fractional derivative $D_{t_{0}, t}^{\alpha}$ is either Caputo or Riemann-Liouville.

\section{Preliminaries and Notations}

We introduce in this section several definitions and results that will be used later in the sequel.

Definition 1 ([19]). The Riemann-Liouville fractional integral of a function $f \in C\left[t_{0}, T\right]$ (or $L^{1}$ ) is defined by

$$
I^{\alpha} f(t)=\frac{1}{\Gamma(\alpha)} \int_{t_{0}}^{t}(t-s)^{\alpha-1} f(s) d s, \quad \alpha>0 .
$$

Definition 2 ([19]). The Riemann-Liouville fractional derivative of order $\alpha \in(n-1, n), n \in \mathbb{N}$, is defined by

$$
{ }^{R L} D_{t_{0}, t}^{\alpha} f(t)=\left(\frac{d}{d t}\right)^{n} I^{n-\alpha} f(t),
$$

where $d / d t$ is the ordinary differential operator.

Theorem 3 ([19]). If $f \in C\left[t_{0}, T\right]$ and $\alpha \in(n-1, n), n \in \mathbb{N}$, then the equality

$$
\begin{array}{r}
I^{\alpha R L} D_{t_{0}, t}^{\alpha} f(t)=f(t)-\sum_{k=1}^{n} \frac{f_{n-\alpha}^{(n-k)}\left(t_{0}\right)}{\Gamma(\alpha-k+1)}\left(t-t_{0}\right)^{\alpha-k}, \\
t>t_{0},
\end{array}
$$

holds everywhere on $\left[t_{0}, T\right]$, where $f_{n-\alpha}(t)=I^{n-\alpha} f(t) \epsilon$ $C^{n}\left[t_{0}, T\right]$.

Definition 4 ([20]). The Caputo derivative (left-sided) of a function $f \in C^{n}\left[t_{0}, T\right], n-1<\alpha \leq n$, is defined as

$$
{ }^{c} D_{t_{0}, t}^{\alpha} f(t)= \begin{cases}I^{n-\alpha} f^{(n)}(t), & n-1<\alpha<n, \\ f^{(n)}(t), & \alpha=n .\end{cases}
$$

Lemma 5 ([21]). Let $n-1<\alpha \leq n$; then

$$
I^{\alpha c} D_{t_{0}, t}^{\alpha} f(t)=f(t)-\sum_{k=0}^{n-1} \frac{f^{(k)}\left(t_{0}\right)}{k !}\left(t-t_{0}\right)^{k} .
$$

Definition 6. The Laplace transform of a real function $f$ is defined as

$$
F(s)=\mathscr{L}\{f\}(s)=\int_{0}^{\infty} e^{-s t} f(t) d t .
$$

The Laplace transform of the Caputo fractional derivative is given by

$$
\begin{aligned}
\mathscr{L}\left\{{ }^{c} D_{t_{0}, t}^{\alpha} f(t)\right\}(s)= & s^{\alpha} \mathscr{L}\{f(t)\}(s) \\
& -\sum_{k=0}^{n-1} s^{\alpha-k-1} f^{(k)}\left(t_{0}\right), \\
& n-1<\alpha<n .
\end{aligned}
$$

The Laplace transform of the Riemann-Liouville fractional derivative is given by [20]

$$
\begin{aligned}
\mathscr{L}\left\{{ }^{R L} D_{t_{0}, t}^{\alpha} f(t)\right\}(s)= & s^{\alpha} L\{f(t)\}(s) \\
& -\sum_{k=0}^{n-1} s^{k R L} D_{t_{0}, t}^{\alpha-k-1} f\left(t_{0}\right), \\
& n-1<\alpha<n .
\end{aligned}
$$

The Laplace transform of $t^{\alpha}$ is given by

$$
\mathscr{L}\left\{t^{\alpha}\right\}=\frac{\Gamma(\alpha+1)}{s^{\alpha+1}}, \quad s>0, \alpha>-1 .
$$

The convolution of the functions $f$ and $g$ is defined as

$$
(f * g)(t)=\int_{0}^{t} g(t-s) f(s) d s .
$$

The inverse Laplace transform of the product of the functions $f$ and $g$ is given by

$$
\begin{aligned}
\mathscr{L}^{-1} & \{f(s) g(s)\}(t) \\
= & \mathscr{L}^{-1}\{f(s)\}(t) * \mathscr{L}^{-1}\{g(s)\}(t) \\
= & \int_{0}^{t} \mathscr{L}^{-1}\{g(s)\}(t-r) \mathscr{L}^{-1}\{f(s)\}(r) d r .
\end{aligned}
$$




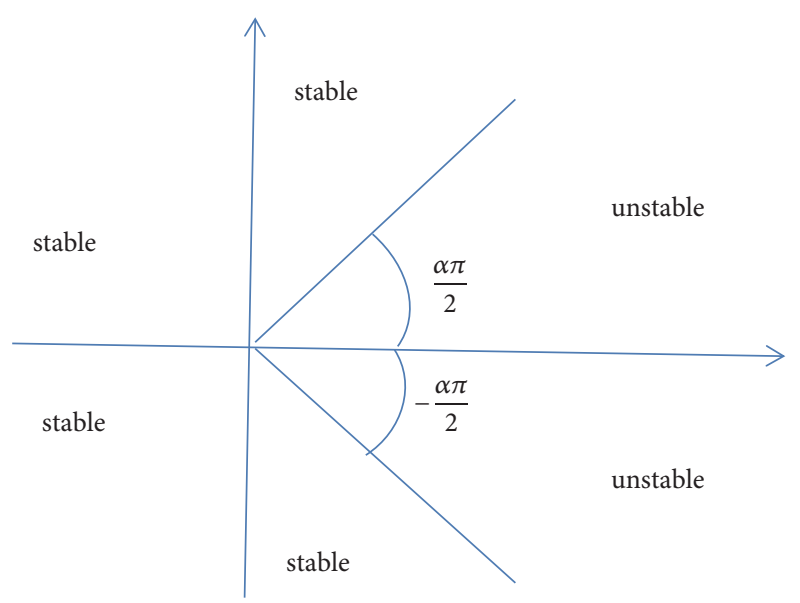

Stability of the system if $0<\alpha<1$

FIGURE 1

Lemma 7 ([20]). The Laplace transform of the Mittag-Leffler function is given by

$$
\begin{aligned}
& \mathscr{L}\left\{t^{\beta-1} E_{\alpha, \beta}\left( \pm \theta t^{\alpha}\right)\right\}(s)=s^{\alpha-\beta}\left(s^{\alpha} \mp \theta\right)^{-1}, \\
& \operatorname{Re}(s)>\theta^{1 / \alpha} .
\end{aligned}
$$

We conclude that

$$
\mathscr{L}\left\{E_{\alpha}\left( \pm \theta t^{\alpha}\right)\right\}(s)=s^{\alpha-1}\left(s^{\alpha} \mp \theta\right)^{-1}, \quad \operatorname{Re}(s)>\theta^{1 / \alpha} .
$$

The Mittag-Leffler function [21] is very important in solving fractional differential equations and in many applications. The exponential function as special case of Mittag-Leffler which is frequently used in the solution of integer order differential equations. The Mittag-Leffler function of one parameter is defined as

$$
E_{\alpha}(z)=\sum_{k=0}^{\infty} \frac{z^{k}}{\Gamma(\alpha k+1)}, \quad z \in \mathbb{C}, \operatorname{Re}(\alpha)>0 .
$$

The Mittag-Leffler function of two parameters is defined by

$$
\begin{aligned}
E_{\alpha, \beta}(z)=\sum_{k=0}^{\infty} \frac{z^{k}}{\Gamma(\alpha k+\beta)}, & \\
z & \in \mathbb{C}, \operatorname{Re}(\alpha)>0, \operatorname{Re}(\beta)>0 .
\end{aligned}
$$

which both satisfy the fact that $E_{\alpha, 1}(z)=E_{\alpha}(z)$, and $E_{1}(z)=$ $e^{z}$.

Lemma 8 ([22]). The system $D_{t_{0}, t}^{\alpha} x(t)=A x(t)$ is asymptotically stable if $|\arg (e i g(A))|>\alpha \pi / 2$ where $0<\alpha<2$, and eig $(A)$ are the eigenvalues of matrix $A$. The stable and unstable regions for $0<\alpha<1$ and $1<\alpha<2$ are shown in Figures 1 and 2, respectively.

Remark 9 ([22]). The perturbed system $D_{t_{0}, t}^{\alpha} x(t)=A x(t)+$ $f(t, x(t))$ is asymptotically stable if $|\arg (e i g(A))|>\alpha \pi / 2$, and $f$ satisfies the fact that $\|f(t, x)\| \leq \gamma(t)\|x\|, t \geq t_{0}$, for some $\gamma \in L^{1}$.

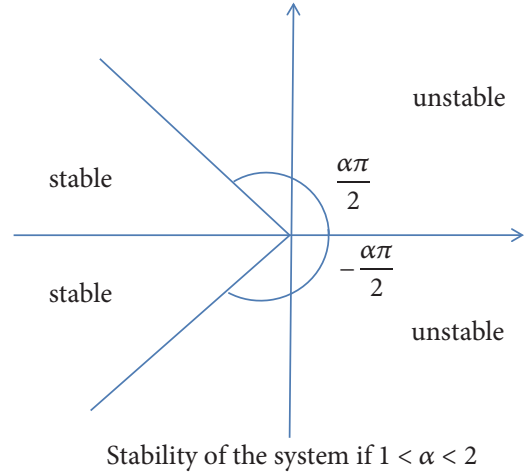

Figure 2

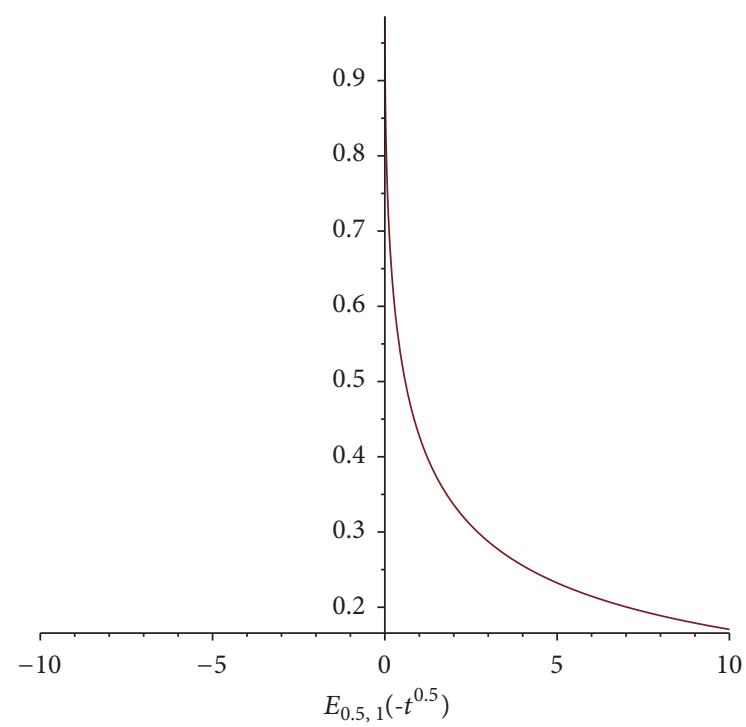

FiguRe 3

It may be observed that the behavior of Mittag-Leffler function is a relaxation for $\alpha<1$, it is exponential for $\alpha=1$, it becomes a damped oscillation for $1<\alpha<2$, and it oscillates for $\alpha=2$. The decay is very fast as $t \longrightarrow 0^{+}$and very slow ast $\longrightarrow \infty$. See Figures 3, 4, and 5 .

Definition 10 ([23]). The constant $x_{e q}$ is an equilibrium point of fractional differential system

$$
D_{t_{0}, t}^{\alpha} x(t)=f(t, x(t)), \quad t \in\left[t_{0}, \infty\right)
$$

if and only if $f\left(t, x_{e q}\right)=\left.D_{t_{0}, t}^{\alpha} x(t)\right|_{x(t)=x_{e q}}$, for all $t>t_{0}$, where $D_{t_{0}, t}^{\alpha}$ means either Caputo or Riemann-Liouville fractional operator.

For convenience, we state all definitions and theorems for stability in Caputo sense when the equilibrium point is the origin of $\mathbb{R}^{n}$, i.e., $x_{e q}=0$. There is no loss of generality in doing so because any equilibrium point can be shifted to the origin via a change of variables. Indeed, suppose that the 


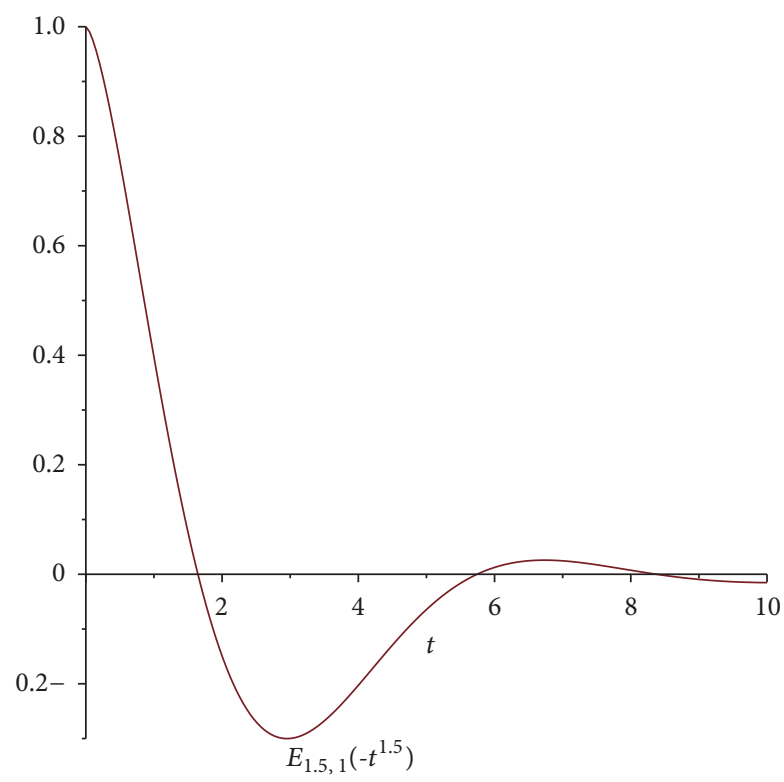

Figure 4

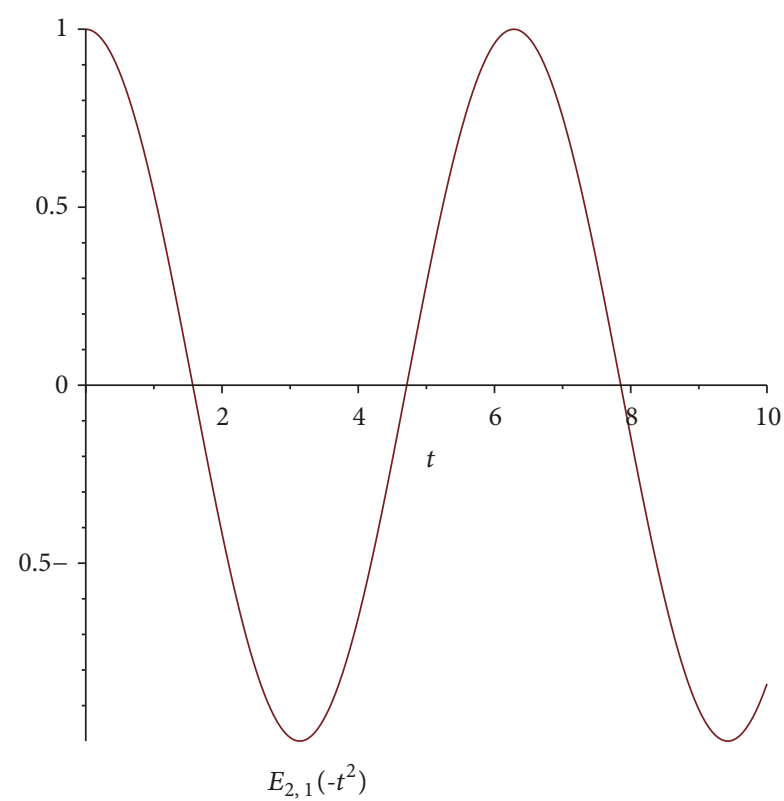

FigURE 5

equilibrium point is $y$, then consider the change of variable $z=x-y$. Therefore, the Caputo derivative of $z$ is

$$
\begin{aligned}
{ }^{c} D_{t_{0}, t}^{\alpha} z & ={ }^{c} D_{t_{0}, t}^{\alpha}(x-y)=f(t, x)=f(t, z+y) \\
& =g(t, y),
\end{aligned}
$$

where $g(t, 0)=0$, and in the new variable $y$, the system has an equilibrium point at the origin.

Definition 11 ([23]). The zero solution of system (22) is said to be stable if there exists $M>0$ such that $\|x(t)\| \leq M$, for all $t>t_{0}$.
Definition 12 ([23]). The zero solution of system (22) is said to be asymptotically stable if $\|x(t)\| \longrightarrow 0$, as $t \longrightarrow \infty$.

A function $m$ is Lipschitz function if there exists a positive constant $\rho$ such that

$$
\|m(x)-m(y)\| \leq \rho\|x-y\| .
$$

Definition 13 ([23]). Let $B \subset \mathbb{R}^{n}$ be a domain containing the origin. The zero solution of system (22) is said to satisfy the Mittag-Leffler criteria if

$$
\|x(t)\| \leq\left(m\left(x\left(t_{0}\right)\right) E_{\alpha, 1}\left(-\theta\left(t-t_{0}\right)^{\alpha}\right)\right)^{\gamma}, \quad t>t_{0},
$$

where $\theta \geq 0, \gamma>0, m(0)=0, m(x) \geq 0,0<\alpha<1$, and $m$ is Lipschitz function on $B$.

Definition 14 ([23]). Let $B \subset \mathbb{R}^{n}$ be a domain containing the origin. The zero solution of system (22) is said to satisfy the generalized Mittag-Leffler criteria if

$$
\begin{array}{r}
\|x(t)\| \\
\leq\left(m\left(x\left(t_{0}\right)\right)\left(t-t_{0}\right)^{-\delta} E_{\alpha, 1-\delta}\left(-\theta\left(t-t_{0}\right)^{\alpha}\right)\right)^{\gamma}, \\
t>t_{0},
\end{array}
$$

where $\theta \geq 0, \gamma>0,0<\alpha<1,-\alpha<\delta<1-\alpha, m(0)=0$, $m(x) \geq 0$, and $m$ is Lipschitz function on $B$.

Remark 15. If $\delta=0$, the generalized Mittag-Leffler is reduced to Mittag-Leffler criteria. Moreover, the MittagLeffler stability implies the asymptotic stability. According to investigations in [17], the above definitions can be applied to the case of $1<\alpha<2$ for some systems.

Definition 16 ([24]). A function $g$ is said to belong to a class$\mathrm{K}$ function if $g:[0, \infty) \longrightarrow[0, \infty)$ is a continuous function satisfying $g(0)=0$, and it is strictly increasing.

Definition 17 ([9]). Let $x^{*}$ be an equilibrium point for system (22), and let $L: O \longrightarrow \mathbb{R}$ defined on an open set $O$ containing $x^{*}$ and $L$ is continuously differentiable on $O$. Suppose further that

$$
\begin{gathered}
L\left(x^{*}\right)=0, \\
L(x)>0,
\end{gathered}
$$

for $x \neq x^{*}$.

Then $x^{*}$ is said to be stable if $\dot{L}(x)=(d / d x) L(x(t)) \leq 0$, on $O \backslash\left\{x^{*}\right\}$.

A function $L$ satisfying the above two conditions is called a Lyapunov function. 
Lemma 18. Let $f:\left[t_{0}, \infty\right) \times \mathbb{R}^{n} \longrightarrow \mathbb{R}^{n}$ be Lipschitz function; then the unique solution of (6) is

$x(t)$

$$
\begin{aligned}
= & E_{\alpha, 1}\left(A\left(t-t_{0}\right)^{\alpha}\right) x_{0} \\
& +\left(t-t_{0}\right) E_{\alpha, 2}\left(A\left(t-t_{0}\right)^{\alpha}\right) x_{1} \\
& +\int_{t_{0}}^{t}(t-\tau)^{\alpha-1} E_{\alpha, \alpha}\left(A(t-\tau)^{\alpha}\right) f(\tau, x(\tau)) d \tau .
\end{aligned}
$$

Proof. The existence and uniqueness of solution for (6) can be obtained by applying Banach fixed point Theorem (see [17]). By applying Laplace transform for (6), we have

$$
\begin{aligned}
\mathscr{L}\left\{{ }^{c} D_{t_{0}, t}^{\alpha} x(t)\right\}(s)= & \mathscr{L}\{A x(t)\}(s) \\
& +\mathscr{L}\{f(t, x(t))\}(s),
\end{aligned}
$$

and, then,

$$
\begin{aligned}
s^{\alpha} & \mathscr{L}\{x(t)\}(s)-s^{\alpha-1} x_{0}-s^{\alpha-2} x_{1} \\
& =\mathscr{L}\{A x(t)\}(s)+\mathscr{L}\{f(t, x(t))\}(s) .
\end{aligned}
$$

Set $\mathscr{L}\{f(t, x(t))\}(s)=F(s, x(s))$; then we get

$$
\mathscr{L}\{x(t)\}(s)\left[s^{\alpha}-A\right]=s^{\alpha-1} x_{0}+s^{\alpha-2} x_{1}+F(s, x(s)),
$$

which implies

$$
\begin{aligned}
\mathscr{L}\{x(t)\}(s)= & s^{\alpha-1}\left[s^{\alpha}-A\right]^{-1} x_{0}+s^{\alpha-2}\left[s^{\alpha}-A\right]^{-1} x_{1} \\
& +\left[s^{\alpha}-A\right]^{-1} F(s, x(s)) .
\end{aligned}
$$

Now, taking Laplace inverse to both sides and using Lemma 7, we obtain

$$
\begin{aligned}
x(t)= & E_{\alpha}\left(A\left(t-t_{0}\right)^{\alpha}\right) x_{0} \\
& +\left(t-t_{0}\right) E_{\alpha, 2}\left(A\left(t-t_{0}\right)^{\alpha}\right) x_{1} \\
& +\mathscr{L}^{-1}\left\{\left[s^{\alpha}-A\right]^{-1} F(s, x(s))\right\}(t) .
\end{aligned}
$$

By convolution theorem of Laplace transform on the third term of (33), we have

$$
\begin{aligned}
\mathscr{L}^{-1} & \left\{\left[s^{\alpha}-A\right]^{-1} F(s, u(s))\right\}(t) \\
& =\mathscr{L}^{-1}\left\{\left[s^{\alpha}-A\right]^{-1}\right\}(t) * \mathscr{L}^{-1}\{F(s, u(s))\}(t) \\
& =\int_{t_{0}}^{t}(t-\tau)^{\alpha-1} E_{\alpha, \alpha}\left(A(t-\tau)^{\alpha}\right) f(\tau, x(\tau)) d \tau .
\end{aligned}
$$

Thus, we get the result.

Lemma 19. Let $V:\left[t_{0}, \infty\right) \times \mathbb{R}^{n} \longrightarrow \mathbb{R}_{+}$be continuously differentiable function. The solution of the fractional differential equation

$$
{ }^{c} D_{t_{0}, t}^{\alpha} V(t, x(t))=-k V(t, x(t))
$$

is

$$
\begin{aligned}
V(t, x(t))= & V_{0} E_{\alpha, 1}\left(-k\left(t-t_{0}\right)^{\alpha}\right) \\
& +V_{1}\left(t-t_{0}\right) E_{\alpha, 2}\left(-k\left(t-t_{0}\right)^{\alpha}\right),
\end{aligned}
$$

where $V_{0}=V\left(t_{0}, x\left(t_{0}\right)\right)$, and $V_{1}=V^{\prime}\left(t_{0}, x\left(t_{0}\right)\right)$.

Proof. By taking Laplace transformation to both sides of (35), we get

$$
\mathscr{L}\left\{{ }^{c} D_{t_{0}, t}^{\alpha} V(t, x(t))\right\}=-k \mathscr{L}\{V(t, x(t))\} .
$$

Then

$$
\begin{aligned}
s^{\alpha} \mathscr{L} & \{V(t, x(t))\}-s^{\alpha-1} V_{0}-s^{\alpha-2} V_{1} \\
& =-k \mathscr{L}\{V(t, x(t))\} .
\end{aligned}
$$

So,

$$
\begin{aligned}
\mathscr{L}\{V(t, x(t))\} & =\frac{s^{\alpha-1} V_{0}+s^{\alpha-2} V_{1}}{s^{\alpha}+k} \\
& =V_{0} \frac{s^{\alpha-1}}{s^{\alpha}+k}+V_{1} \frac{s^{\alpha-2}}{s^{\alpha}+k} .
\end{aligned}
$$

By applying the inverse Laplace transform $\mathscr{L}^{-1}$ and using Lemma 7, we obtain the solution.

Lemma 20. Let $V:\left[t_{0}, \infty\right) \times \mathbb{R}^{n} \longrightarrow \mathbb{R}_{+}$be continuously differentiable function. The solution of the fractional differential equation

$$
{ }^{R L} D_{t_{0}, t}^{\alpha} V(t, x(t))=-M(t),
$$

is

$$
\begin{aligned}
V(t, x(t))= & \frac{V_{0}}{\Gamma(\alpha)}\left(t-t_{0}\right)^{\alpha-1}+\frac{V_{1}}{\Gamma(\alpha-1)}\left(t-t_{0}\right)^{\alpha-2} \\
& -\frac{1}{\Gamma(\alpha)} \int_{t_{0}}^{t}(t-s)^{\alpha-1} M(s) d s
\end{aligned}
$$

where $\left.{ }^{R L} D_{t_{0}, t}^{\alpha-m} V(t, x(t))\right|_{t=t_{0}+}=V_{m-1}, m=1,2$.

Proof. By taking Laplace transform to both sides of (40), we get

$$
s^{\alpha} \mathscr{L}\{V(t, x(t))\}-V_{0}-s V_{1}=-\mathscr{L}\{M(t)\},
$$

which implies that

$$
\mathscr{L}\{V(t, x(t))\}=V_{0} \frac{1}{s^{\alpha}}+V_{1} \frac{1}{s^{\alpha-1}}-\frac{1}{s^{\alpha}} \mathscr{L}\{M(t)\} .
$$

By taking $\mathscr{L}^{-1}$ for both sides, we obtain

$$
\begin{aligned}
V(t, x(t))= & \frac{V_{0}}{\Gamma(\alpha)}\left(t-t_{0}\right)^{\alpha-1}+\frac{V_{1}}{\Gamma(\alpha-1)}\left(t-t_{0}\right)^{\alpha-2} \\
& -\mathscr{L}^{-1}\left\{\frac{1}{s^{\alpha}}\right\} * M(t) \\
= & \frac{V_{0}}{\Gamma(\alpha)}\left(t-t_{0}\right)^{\alpha-1}+\frac{V_{1}}{\Gamma(\alpha-1)}\left(t-t_{0}\right)^{\alpha-2} \\
& -\frac{1}{\Gamma(\alpha)} \int_{t_{0}}^{t}(t-r)^{\alpha-1} M(r) d r .
\end{aligned}
$$

This finishes the proof. 
Remark 21. In Lemma 20, if we replace Riemann-Liouville derivative by Caputo derivative, then the solution is

$$
\begin{aligned}
V(t, x(t))= & V_{0}+V_{1}\left(t-t_{0}\right) \\
& -\frac{1}{\Gamma(\alpha)} \int_{t_{0}}^{t}(t-s)^{\alpha-1} M(s) d s,
\end{aligned}
$$

where $V_{0}=V\left(t_{0}, x\left(t_{0}\right)\right)$, and $V_{1}=V^{\prime}\left(t_{0}, x\left(t_{0}\right)\right)$.

Lemma 22. Let $V:\left[t_{0}, \infty\right) \times \mathbb{R}^{n} \longrightarrow \mathbb{R}_{+}$be continuously differentiable function. The solution of the fractional differential equation

$$
{ }^{R L} D_{t_{0}, t}^{\alpha} V(t, x(t))=-k V(t, x(t)), \quad k \geq 0,
$$

is

$$
\begin{aligned}
V(t, x(t))= & V_{0}\left(t-t_{0}\right)^{\alpha-1} E_{\alpha, \alpha}\left(-k\left(t-t_{0}\right)^{\alpha}\right) \\
& +V_{1}\left(t-t_{0}\right)^{\alpha-2} E_{\alpha, \alpha-1}\left(-k\left(t-t_{0}\right)^{\alpha}\right),
\end{aligned}
$$

where $\left.{ }^{R L} D_{t_{0}, t}^{\alpha-m} V(t, x(t))\right|_{t=t_{0}+}=V_{m-1}(m=1,2)$.

Proof. By taking Laplace transform for both sides of (46), we get

$$
\mathscr{L}\left\{{ }^{R L} D_{t_{0}, t}^{\alpha} V(t, x(t))\right\}=-k \mathscr{L}\{V(t, x(t))\},
$$

and then

$$
s^{\alpha} \mathscr{L}\{V(t, x(t))\}-V_{0}-s V_{1}=-k \mathscr{L}\{V(t, x(t))\} .
$$

So,

$$
\mathscr{L}\{V(t, x(t))\}=\frac{V_{0}+s V_{1}}{s^{\alpha}+k}=V_{0} \frac{1}{s^{\alpha}+k}+V_{1} \frac{s}{s^{\alpha}+k} .
$$

By taking $\mathscr{L}^{-1}$ and using Lemma 7, we obtain the results.

\section{Stability Results}

In this section, we study the stability of nonlinear fractional differential systems with Caputo derivative by utilizing a Lyapunov-like function, taking into account the relation between asymptotical stability and generalized Mittag-Leffler criteria.

The Lyapunov direct method is a sufficient condition to show the stability of systems, which means the system may still be stable even if one cannot find a Lyapunov function candidate to conclude the system stability property.

Hereafter, we assume that the Lyapunov function $V$ : $\left[t_{0}, \infty\right) \times \mathbb{R}^{n} \longrightarrow \mathbb{R}_{+}$is continuously differentiable with respect to $t$, Lipschitz with respect to $x$, and $V(t, 0)=0$.

Theorem 23. Let $x=0$ be an equilibrium point of system (6) and let $V$ satisfy

$$
a\|x\|^{b} \leq V(t, x(t)),
$$

and

$$
{ }^{R L} D_{t_{0}, t}^{\alpha} V(t, x(t)) \leq 0,
$$

where $x \in \mathbb{R}^{n}, a$, and $b$ are positive real numbers. Then, the zero solution is asymptotically stable whenever $\left.{ }^{R L} D_{t_{0}, t}^{\alpha-1} V(t, x(t))\right|_{t=t_{0}+}=0$, and $\left.{ }^{R L} D_{t_{0}, t}^{\alpha-2} V(t, x(t))\right|_{t=t_{0}+} \geq 0$.

Proof. Inequality (52) implies the existence of a nonnegative function $M$ such that

$$
{ }^{R L} D_{t_{0}, t}^{\alpha} V(t, x(t))=-M(t) .
$$

Using Lemma 20, we get

$$
\begin{aligned}
V(t, x(t))= & \frac{V_{0}}{\Gamma(\alpha)}\left(t-t_{0}\right)^{\alpha-1}+\frac{V_{1}}{\Gamma(\alpha-1)}\left(t-t_{0}\right)^{\alpha-2} \\
& -\frac{1}{\Gamma(\alpha)} \int_{t_{0}}^{t}(t-s)^{\alpha-1} M(s) d s
\end{aligned}
$$

where $\left.{ }^{R L} D_{t_{0}, t}^{\alpha-m} V(t, x(t))\right|_{t=t_{0}+}=V_{m-1}(m=1,2)$. The negativity of the last term of (54) will imply that

$$
V(t, x(t)) \leq \frac{V_{0}}{\Gamma(\alpha)}\left(t-t_{0}\right)^{\alpha-1}+\frac{V_{1}}{\Gamma(\alpha-1)}\left(t-t_{0}\right)^{\alpha-2}
$$

In accordance with (51), we obtain

$$
\begin{aligned}
& \|x(t)\| \\
& \quad \leq\left\{\frac{V_{0}}{a \Gamma(\alpha)}\left(t-t_{0}\right)^{\alpha-1}+\frac{V_{1}}{a \Gamma(\alpha-1)}\left(t-t_{0}\right)^{\alpha-2}\right\}^{1 / b} .
\end{aligned}
$$

Now, set $V_{0}=0$; then $\|x(t)\| \longrightarrow 0$, as $t \longrightarrow \infty$. Therefore, the zero solution of system (6) is asymptotically stable.

Theorem 24. Let $x=0$ be an equilibrium point of system (6) and let $V$ satisfy

$$
\begin{aligned}
a\|x\|^{b} & \leq V(t, x(t)), \\
{ }^{c} D_{t_{0}, t}^{\alpha} V(t, x(t)) & \leq-c V(t, x(t)),
\end{aligned}
$$

where $x \in \mathbb{R}^{n}, a, b$, and c are arbitrary positive constants. Then, the zero solution satisfies the Mittag-Leffler criteria whenever $V\left(t_{0}, x\left(t_{0}\right)\right) \geq 0$, and $V^{\prime}\left(t_{0}, x\left(t_{0}\right)\right)=0$.

Proof. Using (58), there exists a nonnegative function $M$ such that

$$
{ }^{c} D_{t_{0}, t}^{\alpha} V(t, x(t))+c V(t, x(t))=-M(t) .
$$

By Lemma 18 and noting that $I^{\alpha} M(t)$ is nonnegative, we deduce that

$$
\begin{aligned}
V(t, x(t)) \leq & V_{0} E_{\alpha, 1}\left(-c\left(t-t_{0}\right)^{\alpha}\right) \\
& +V_{1}\left(t-t_{0}\right) E_{\alpha, 2}\left(-c\left(t-t_{0}\right)^{\alpha}\right),
\end{aligned}
$$

where $V_{0}=V\left(t_{0}, x\left(t_{0}\right)\right)$, and $V_{1}=V^{\prime}\left(t_{0}, x\left(t_{0}\right)\right)=0$. Then,

$$
V(t, x(t)) \leq V_{0} E_{\alpha, 1}\left(-c\left(t-t_{0}\right)^{\alpha}\right) \text {. }
$$


In accordance with (57), we obtain $\|x(t)\| \leq\left[\left(V_{0} / a\right) E_{\alpha, 1}(-\right.$ $\left.\left.c\left(t-t_{0}\right)^{\alpha}\right)\right]^{1 / b}$, for $m=V_{0} / a \geq 0$. Then, the zero solution of system (6) satisfies the Mittag-Leffler criteria.

Theorem 25. Let $x=0$ be an equilibrium point of system (6) and let $V$ satisfy

$$
V(t, x(t)) \geq a\|x\|^{b},
$$

and

$$
{ }^{R L} D_{t_{0}, t}^{\alpha} V(t, x(t)) \leq 0,
$$

where $t \geq t_{0}, x \in \mathbb{R}^{n}, a$ and $b$ are arbitrary positive constant. Then, the zero solution satisfies the generalized Mittag-Leffler criteria whenever $\left.{ }^{R L} D_{t_{0}, t}^{\alpha-1} V(t, x(t))\right|_{t=t_{0}+}=0$, and $\left.{ }^{R L} D_{t_{0}, t}^{\alpha-2} V(t, x(t))\right|_{t=t_{0}+} \geq 0$.

Proof. There exists a nonnegative function $M$ such that

$$
{ }^{R L} D_{t_{0}, t}^{\alpha} V(t, x(t))=-M(t)
$$

By using Lemma 20, we can deduce as in the proof of proceeding results that

$$
\begin{aligned}
V(t, x(t)) \leq & \frac{V_{0}}{\Gamma(\alpha)}\left(t-t_{0}\right)^{\alpha-1} \\
& +\frac{V_{1}}{\Gamma(\alpha-1)}\left(t-t_{0}\right)^{\alpha-2},
\end{aligned}
$$

where $\left.{ }^{R L} D_{t_{0}, t}^{\alpha-m} V(t, x(t))\right|_{t=t_{0}+}=V_{m-1},(m=1,2), 1<\alpha<2$. Therefore,

$$
\begin{aligned}
& \|x(t)\| \\
& \quad \leq\left[\frac{V_{0}}{a \Gamma(\alpha)}\left(t-t_{0}\right)^{\alpha-1}+\frac{V_{1}}{a \Gamma(\alpha-1)}\left(t-t_{0}\right)^{\alpha-2}\right]^{1 / b} .
\end{aligned}
$$

Now, set $V_{0}=0$; then

$$
\begin{aligned}
\|x(t)\| & \leq\left[\frac{V_{1}}{a \Gamma(\alpha-1)}\left(t-t_{0}\right)^{\alpha-2}\right]^{1 / b} \\
& \leq\left[\frac{V_{0}}{a \Gamma(\alpha)}\left(t-t_{0}\right)^{\alpha-1} E_{\alpha, \alpha}\left(0 .\left(t-t_{0}\right)\right]^{1 / b} .\right.
\end{aligned}
$$

Now, set $m=V_{0} / a \Gamma(\alpha-1) \geq 0$, and, therefore, the zero solution of system (6) satisfies the generalized Mittag-Leffler criteria.

Theorem 26. Let $x=0$ be an equilibrium point of system (6). Assume there exists a class-K function g satisfying

$$
\begin{aligned}
& V(t, x(t)) \geq g^{-1}(\|x(t)\|), \\
& { }^{c} D_{t_{0}, t}^{\alpha} V(t, x(t)) \leq 0, \quad t \geq t_{0}, \\
& \sup _{t \geq t_{0}} g\left(V\left(t_{0}, x\left(t_{0}\right)\right)+V^{\prime}\left(t_{0}, x\left(t_{0}\right)\right)\left(t-t_{0}\right)\right) \leq M,
\end{aligned}
$$

for some nonnegative constant $M$. Then, the zero solution is stable.
Proof. Using (69), there exists a nonnegative function $M$ such that ${ }^{c} D_{t_{0}, t}^{\alpha} V(t, x(t))=-M(t)$. Then

$$
V(t, x(t))=V_{0}+V_{1}\left(t-t_{0}\right)-I^{\alpha} M(t),
$$

where $V_{0}=V\left(t_{0}, x\left(t_{0}\right)\right)$, and $V_{1}=V^{\prime}\left(t_{0}, x\left(t_{0}\right)\right)$. Now, (68) and (71) imply that

$$
\begin{aligned}
g^{-1}(\|x(t)\|) & \leq V_{0}+V_{1}\left(t-t_{0}\right)-I^{\alpha} M(t) \\
& \leq V_{0}+V_{1}\left(t-t_{0}\right)
\end{aligned}
$$

Therefore,

$$
\|x(t)\| \leq g\left(V_{0}+V_{1}\left(t-t_{0}\right)\right) .
$$

Then, by (70), we have $\|x(t)\| \leq M, t \geq t_{0}$. Therefore, the zero solution of system (6) is stable.

Theorem 27. Let $x=0$ be an equilibrium point of system (6) and assume that there exists a class-Kfunction $g$ satisfying

$$
\begin{aligned}
V(t, x(t)) & \geq g^{-1}(\|x(t)\|), \\
{ }^{R L} D_{t_{0}, t}^{\alpha} V(t, x(t)) & \leq 0,
\end{aligned}
$$

where $t \geq t_{0}$. Then, the zero solution is asymptotically stable whenever $\left.{ }^{R L} D_{t_{0}, t}^{\alpha-1} V(t, x(t))\right|_{t=t_{0}+}=0$.

Proof. As in Theorem 23, we have

$$
\begin{aligned}
V(t, x(t)) \leq & \frac{V_{0}}{\Gamma(\alpha)}\left(t-t_{0}\right)^{\alpha-1} \\
& +\frac{V_{1}}{\Gamma(\alpha-1)}\left(t-t_{0}\right)^{\alpha-2},
\end{aligned}
$$

where $\left.{ }^{R L} D_{t_{0}, t}^{\alpha-m} V(t, x(t))\right|_{t=t_{0}+}=V_{m-1}(m=1,2)$. Now, inequalities (74) and (76) imply that

$$
\begin{aligned}
& \|x(t)\| \\
& \quad \leq g\left(\frac{V_{0}}{\Gamma(\alpha)}\left(t-t_{0}\right)^{\alpha-1}+\frac{V_{1}}{\Gamma(\alpha-1)}\left(t-t_{0}\right)^{\alpha-2}\right) .
\end{aligned}
$$

Therefore $\|x(t)\| \leq g\left(\left(V_{1} / \Gamma(\alpha-1)\right)\left(t-t_{0}\right)^{\alpha-2}\right)$, for $V_{0}=0$. Hence, the continuity of $g$ implies that $\|x(t)\| \longrightarrow 0$ as $t \longrightarrow$ $\infty$. Therefore, the zero solution of system (6) is asymptotically stable.

\section{The Comparison Results on Stability}

In this section we discuss the comparison of the stability for the two systems with Caputo and Riemann-Liouville derivatives. It is well known that the comparison method is an effective way in judging the stability of ordinary differential systems. We will discuss similar results on the stability of fractional differential systems by using the comparison method. 
Consider the semilinear system

$$
\begin{gathered}
{ }^{R L} D_{t_{0}, t}^{\alpha} u(t)=A u(t)+h(t, u(t)), \\
t>t_{0}, 1<\alpha<2, \\
{ }^{R L} D_{t_{0}, t}^{\alpha-k} u\left(t_{0}+\right) \mid=u_{k-1}, \quad(k=1,2),
\end{gathered}
$$

where $u(t) \in \mathbb{R}^{n}, u_{k} \in \mathbb{R}^{n}(k=0,1), A \in \mathbb{R}^{n \times n}$, and $h:\left[t_{0}, \infty\right) \times \mathbb{R}^{n} \longrightarrow \mathbb{R}^{n}$ is a continuous function such that $h(t, 0)=0$. Moreover, $h$ satisfies the Lipschitz condition with respect to $u$ to ensure the existence and uniqueness of solution (78).

Applying the Laplace technique as in Lemma 18 but using (14), we deduce the following result.

Lemma 28. For $1<\alpha<2$, the solution of system (78) is

$$
\begin{aligned}
& u(t) \\
& =\left(t-t_{0}\right)^{\alpha-1} E_{\alpha, \alpha}\left(A\left(t-t_{0}\right)^{\alpha}\right) u_{0} \\
& \quad+\left(t-t_{0}\right)^{\alpha-2} E_{\alpha, \alpha-1}\left(A\left(t-t_{0}\right)^{\alpha}\right) u_{1} \\
& \quad+\int_{t_{0}}^{t}(t-\tau)^{\alpha-1} E_{\alpha, \alpha}\left(A(t-\tau)^{\alpha}\right) h(\tau, u(\tau)) d \tau
\end{aligned}
$$

In the next result, we recall that the set of vectors can be partially ordered if their components are so.

Theorem 29. Let $u=0$ be an equilibrium of system (78). Assume that $g$ is a class-K function and there exists a function $V:\left[t_{0}, \infty\right) \times \mathbb{R}^{n} \longrightarrow \mathbb{R}^{n}$ such that $V(t, 0)=0$, and for each $t \geq t_{0}$, the following conditions hold:

(i) $\|V(t, u(t))\| \geq,\|V(t, x(t))\| \geq g^{-1}(\|x(t)\|)$, for any solution $x$ of system (6).

(ii) ${ }^{R L} D_{t_{0}, t}^{\alpha} V(t, u(t))-A V(t, u(t)) \leq 0$.

(iii) $\int_{t_{0}}^{t}(t-\tau)^{\alpha-1} E_{\alpha, \alpha}\left(A(t-\tau)^{\alpha}\right) h(\tau, u(\tau)) d \tau$ has nonnegative finite components.

(iv) ${ }^{R L} D_{t_{0}, t}^{\alpha-k} u\left(t_{0}+\right)=\left.{ }^{R L} D_{t_{0}, t}^{\alpha-k} V(t, u(t))\right|_{t=t_{0}+}, k=1,2$.

Moreover, assume that $g$ is nondecreasing. Then

(1) if the zero solution of system (78) is stable, then the zero solution of system (6) is stable;

(2) if the zero solution of system (78) is asymptotically stable, then the zero solution of the system (6) is asymptotically stable.

Proof. (1) Let $M(t)$ be vector-valued function defined on $\mathbb{R}^{n}$ whose components are nonnegative and by (ii) it satisfies the following:

$$
{ }^{R L} D_{t_{0}, t}^{\alpha} V(t, u(t))=A V(t, u(t))-M(t) .
$$

As in the proof of Theorem 24, we have

$$
\begin{aligned}
V(t, u(t))= & \left(t-t_{0}\right)^{\alpha-1} E_{\alpha, \alpha}\left(A\left(t-t_{0}\right)^{\alpha}\right) V_{0} \\
& +\left(t-t_{0}\right)^{\alpha-2} E_{\alpha, \alpha-1}\left(A\left(t-t_{0}\right)^{\alpha}\right) V_{1}
\end{aligned}
$$

$$
-I^{\alpha} M(t)
$$

$$
\begin{aligned}
\leq & \left(t-t_{0}\right)^{\alpha-1} E_{\alpha, \alpha}\left(A\left(t-t_{0}\right)^{\alpha}\right) V_{0} \\
& +\left(t-t_{0}\right)^{\alpha-2} E_{\alpha, \alpha-1}\left(A\left(t-t_{0}\right)^{\alpha}\right) V_{1},
\end{aligned}
$$

where $V_{0}=\left.{ }^{R L} D_{t_{0}, t}^{\alpha-1} V(t, u(t))\right|_{t=t_{0}+}$, and $V_{1}={ }^{R L} D_{t_{0}, t}^{\alpha-2} V(t$, $u(t))\left.\right|_{t=t_{0+}}$. Using condition (iv), we have $u_{0}=V_{0}$, and $u_{1}=$ $V_{1}$; hence condition (iii) implies that

$$
\begin{aligned}
V(t, u(t)) \\
\leq\left(t-t_{0}\right)^{\alpha-1} E_{\alpha, \alpha}\left(A\left(t-t_{0}\right)^{\alpha}\right) u_{0} \\
\quad+\left(t-t_{0}\right)^{\alpha-2} E_{\alpha, \alpha-1}\left(A\left(t-t_{0}\right)^{\alpha}\right) u_{1} \\
\quad+\int_{t_{0}}^{t}(t-\tau)^{\alpha-1} E_{\alpha, \alpha}\left(A(t-\tau)^{\alpha}\right) h(\tau, u(\tau)) d \tau \\
=u(t) .
\end{aligned}
$$

We deduce that

$$
\|V(t, u(t))\| \leq\|u(t)\| .
$$

Now, condition (i) implies that

$$
\begin{aligned}
g^{-1}(\|x(t)\|) & \leq\|V(t, x(t))\| \leq\|V(t, u(t))\| \\
& \leq\|u(t)\| .
\end{aligned}
$$

Since, the zero solution of system (78) is stable, then there exists $M>0$ such that

$$
g^{-1}(\|x(t)\|) \leq\|u(t)\| \leq M
$$

which implies that $\|x(t)\| \leq g(M)=L, L>0$. Therefore, the zero solution of system (6) is stable.

(2) Let the zero solution of system (78) be asymptotically stable, then $\lim _{t \rightarrow \infty}\|u(t)\|=0$. Again, using $g^{-1}(\|x(t)\|) \leq$ $\|u(t)\|$, we deduce that $0 \leq\|x(t)\| \leq g(\|u(t)\|)$. With the continuity of $g$ and the condition $g(0)=0$, we have $\lim _{t \rightarrow \infty}\|x(t)\|=0$; then the zero solution of system (6) is asymptotically stable.

\section{Applications}

The Hopfield neural networks with constant external inputs are described by [4]

$$
{ }^{c} D_{0, t^{+}}^{1^{+}} x_{i}(t)=-a_{i} x_{i}(t)+\sum_{j=1}^{n} b_{i j} f_{j}\left(x_{j}(t)\right)+I_{i},
$$

where $i=1,2, \ldots, n$ and $n$ is the number of units in a neural network, $x_{i}(t)$ is the state of the $i$ th unit at time $t, f_{j}$ denotes the activation function of the $j$ th neuron, $b_{i j}$ denotes the constant connection weight of the $j$ th neuron on the $i$ th neuron, $a_{i}>0$ represents the rate with which the $i$ th neuron resets its potential to the resting state when disconnected 
from the network, $I_{i}$ denotes the constant external inputs, and $1^{+}$means the approach of the fractional order $\alpha$ to classical first-order derivative from right side. System (6) can be easily obtained by letting $x=\left[x_{i}\right]_{n \times 1}, A=\left[-a_{i j}\right]_{n \times n}$, where $a_{i j}=a_{i}$, for $i=j$ and $a_{i j}=0$, for $i \neq j$, and $f=\left[f_{i}\right]_{n \times 1}$, where $f_{i}(t, x(t))=\sum_{j=1}^{n} b_{i j} f_{j}\left(x_{j}(t)\right)+I_{i}, i=1,2, \ldots, n$.

Example 30. Consider the scalar Hopfield neural networks system

$$
{ }^{c} D_{0, t}^{\alpha}|x(t)|=-|x(t)|+f(t, x(t)),
$$

$$
t \geq 0,1<\alpha<2,
$$

for $x(0)=0=x^{\prime}(0)$. Assume that the Lyapunov function is $V(t, x(t))=|x(t)|$ and that $f(t, x(t)) \leq 0, f(t, 0)=0$; then,

$$
\begin{aligned}
{ }^{c} D_{0, t}^{\alpha} V(t, x(t)) & =-V(t, x(t))+f(t, x(t)) \\
& \leq-V(t, x(t)) .
\end{aligned}
$$

Thus, the conditions of Theorem 24 hold with $a=b=c=$ 1. Therefore, the zero solution of system (87) satisfies the Mittag-Leffler criteria.

To illustrate the biological applications [18], we consider two habitat areas, with a fish population dispersing between the two areas, whilst fishing takes place only in region 2, with region 1 established as a no-fishing zone. To describe the ecological linkage between the reserve and fishing ground we propose the fractional alternative model of the autonomous linear system

$$
\begin{aligned}
& { }^{c} D_{0, t}^{\alpha} x_{1}(t)=-\left(m_{1}+D_{1}\right) x_{1}(t)+D_{2} x_{2}(t), \\
& { }^{c} D_{0, t}^{\alpha} x_{2}(t)=-\left(m_{2}+D_{2}+E\right) x_{2}(t)+D_{1} x_{1}(t) .
\end{aligned}
$$

Here, $m_{i}>0$ is the natural mortality (death) rate, $D_{i}>0$ is the dispersal rate, and $E>0$ is the harvesting rate. If we assume that $x=\left[x_{1}, x_{2}\right]^{T}$, and $A=\left[\begin{array}{cc}-\left(m_{1}+D_{1}\right) & D_{2} \\ D_{1} & -\left(m_{2}+D_{2}+E\right)\end{array}\right]$, we obtain the autonomous system

$$
{ }^{c} D_{0, t}^{\alpha} x(t)=A x(t)
$$

The corresponding nonautonomous system can be modeled as (6).

Example 31. Consider the system

$$
\begin{aligned}
& { }^{R L} D_{0, t}^{1.5} x_{1}(t)=-x_{1}(t)+0.5 x_{2}(t), \\
& { }^{R L} D_{0, t}^{1.5} x_{2}(t)=x_{1}(t)-x_{2}(t)+\frac{e^{-t}\left|x_{2}(t)\right|}{\left|x_{1}(t)\right|+1},
\end{aligned}
$$

for $t>0$, and $x(0)=0, x^{\prime}(0)=1$. Let $A=\left(\begin{array}{cc}-1 & 0.5 \\ 0.5 & -1\end{array}\right) \in \mathbb{R}^{2 \times 2}$, and $f(t, x(t))=\left(\begin{array}{c}0 \\ e^{-t}\left|x_{2}(t)\right| /\left(\left|x_{1}(t)\right|+1\right)\end{array}\right) \in \mathbb{R}^{2}, t \geq 0$; then system (91) can be transformed as ${ }^{R L} D_{0, t}^{1.5} x(t)=A x(t)+f(t$, $x(t)$ ), where $x(t)=\left(\begin{array}{l}x_{1}(t) \\ x_{2}(t)\end{array}\right) \in \mathbb{R}^{2}$. The function $f$ is Lipschitz function which ensures the existence of a solution $x(t)$ given by Lemma 28, and $f(t, 0)=0$ ensures the existence of zero solution for (91). The argument of eigenvalues of $A$ is $\pi$; then the solution of (91) is stable (see [17], Theorem 5.1). Therefore, we can always find a constant $M>0$, such that $M^{-2} t^{-4} \leq\|x(t)\| \leq M$, for any $t>0$. Choose the Lyapunov candidate as $V(t, x)=-M t^{2}|x|$; then $V(t, 0)=0,{ }^{R L} D_{0, t}^{1.5} V(t, x)=-M(\Gamma(3) / \Gamma(1.5)) \sqrt{t}|x| \leq 0$, and $\left.{ }^{R L} D_{t_{0}, t}^{0.5} V(t, x)\right|_{t=0+}=-M(\Gamma(3) / \Gamma(2.5)) \sqrt{t^{3}}|x|_{t=0+}=0$. If $g(t)=t^{2}, t \geq 0$, then $g^{-1}(t)=\sqrt{t}$. It is obvious now that $(V(t, x(t)))^{2}=M^{2} t^{4}\|x\|^{2} \geq\|x\|$. Hence all hypotheses of Theorem 27 are satisfied; then system (91) is asymptotically stable.

\section{Data Availability}

All data generated or analyzed during this study are included in this published article.

\section{Conflicts of Interest}

The authors declare that they have no conflicts of interest.

\section{References}

[1] J. Sabatier, O. P. Agrawal, and J. A. Machado, Advance in Fractional Calculus: Theoretical Developments and Applications in Physics and Engineering, Springer, Dordrecht, The Netherlands, 2007.

[2] R. Hilfer, Applications of fractional calculus in physics, World Scientific Publishing Co., Inc, River Edge, NJ, USA, 2000.

[3] X.-J. Wen, Z.-M. Wu, and J.-G. Lu, "Stability analysis of a class of nonlinear fractional-order systems," IEEE Transactions on Circuits and Systems II: Express Briefs, vol. 55, no. 11, pp. 1178$1182,2008$.

[4] E. Kaslik and S. Sivasundaram Seenith, "Nonlinear dynamics and chaos in fractional-order neural networks," Neural Networks, vol. 32, pp. 245-256, 2012.

[5] B. Ahmad, M. M. Matar, and O. M. El-Salmy, "Existence of solutions and Ulam stability for Caputo type sequential fractional differential equations for order $\alpha \in(2,3)$," International Journal of Analysis and Applications, vol. 15, no. 1, pp. 86-101, 2017.

[6] D. Matignon, "Stability results for fractional differential equations with applications to control processing," Proceedings of the IMACS-SMC, vol. 2, pp. 963-968, 1996.

[7] W. Deng, C. Li, and Q. Guo, "Analysis of fractional differential equations with multi-orders," Fractals. Complex Geometry, Patterns, and Scaling in Nature and Society, vol. 15, no. 2, pp. 173182, 2007.

[8] F. Zhang, C. Li, and Y. Q. Chen, "Asymptotical Stability of Nonlinear Fractional Differential System with Caputo Derivative," International Journal of Differential Equations, vol. 2011, Article ID 635165, 12 pages, 2011.

[9] A. Eshkaftaki, J. Alidousti, and R. Khoshsiar Ghaziani, "Stability analysis of fractional-order nonlinear systems via Lyapunov method," Journal of Mahani Mathematical Research Center, vol. 3, no. 1, pp. 61-73, 2014.

[10] J. Sabatier, M. Moze, and C. Farges, "LMI stability conditions for fractional order systems," Computers \& Mathematics with Applications, vol. 59, no. 5, pp. 1594-1609, 2010. 
[11] L. G. Zhang, J. M. Li, and G. P. Chen, "Extension of Lyapunov second method by fractional calculus," Pure and Applied Mathematics, no. 3, 2005.

[12] K. Diethelm and N. J. Ford, "Analysis of fractional differential equations," Journal of Mathematical Analysis and Applications, vol. 265, no. 2, pp. 229-248, 2002.

[13] V. D. Gejji and A. Babakhani, "Analysis of a system of fractional differential equations," Journal of Mathematical Analysis and Applications, vol. 293, no. 2, pp. 511-522, 2004.

[14] Y. Li, Y. Chen, and I. Podlubny, "Mittag-Leffler stability of fractional order nonlinear dynamic systems," Automatica, vol. 45, no. 8, pp. 1965-1969, 2009.

[15] W. Deng, C. Li, and J. Lu, "Stability analysis of linear fractional differential system with multiple time delays," Nonlinear Dynamics, vol. 48, no. 4, pp. 409-416, 2007.

[16] Z. M. Odibat, "Analytic study on linear systems of fractional differential equations," Computers \& Mathematics with Applications, vol. 59, no. 3, pp. 1171-1183, 2010.

[17] F. Zhang and C. Li, "Stability Analysis of Fractional Differential Systems with Order Lying in $(1,2)$," International Journal of Differential Equations, vol. 2011, Article ID 213485, 17 pages, 2011.

[18] L. Idels and M. Kipnis, "Stability criteria for a nonlinear nonautonomous system with delays," Applied Mathematical Modelling: Simulation and Computation for Engineering and Environmental Systems, vol. 33, no. 5, pp. 2293-2297, 2009.

[19] S. Samko, A. Kilbas, and O. Marichev, Fractional Integrals and Derivatives: Theory and Applications, Gordon and Breach, New York, NY, USA, 1993.

[20] I. Podlubny, Fractional Differential Equations, Academic Press, San Diego, CA, USA, 1999.

[21] A. A. Kilbas, H. M. Srivastava, and J. J. Trujillo, Theory and applications of fractional differential equations, Elsevier, Amsterdam, Netherlands, 2006.

[22] S. Priyadharsini, "Stability of fractional neutral and integrodifferential systems," Journal of Fractional Calculus and Applications, vol. 7, no. 1, pp. 87-102, 2016.

[23] Y. Li, Y. Chen, and I. Podlubny, "Stability of fractional-order nonlinear dynamic systems: Lyapunov direct method and generalized Mittag-Leffler stability," Computers \& Mathematics with Applications, vol. 59, no. 5, pp. 1810-1821, 2010.

[24] L. Huang, Stability Theory, Peking University Press, Beijing, China, 1992. 


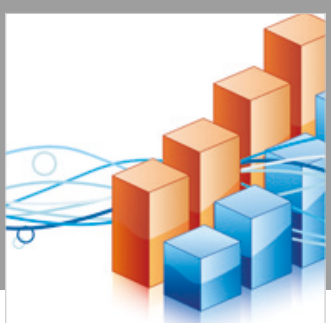

Advances in

Operations Research

\section{-n-m}
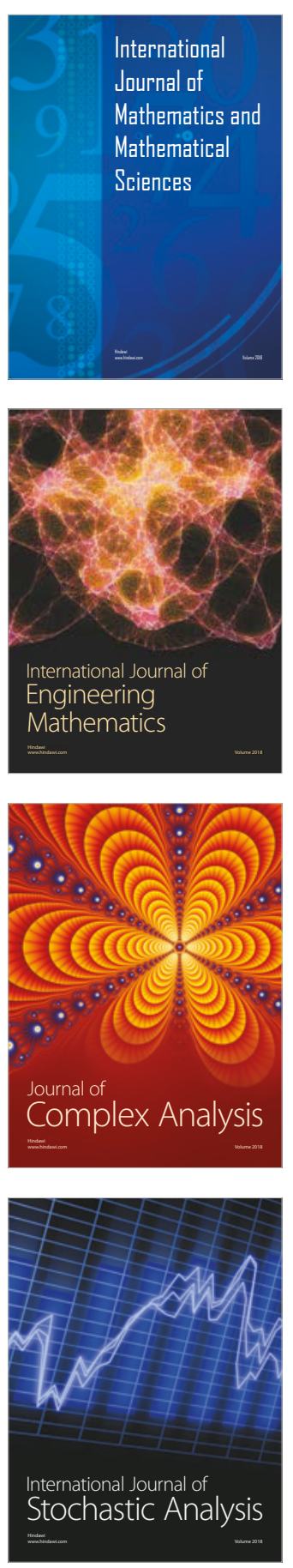
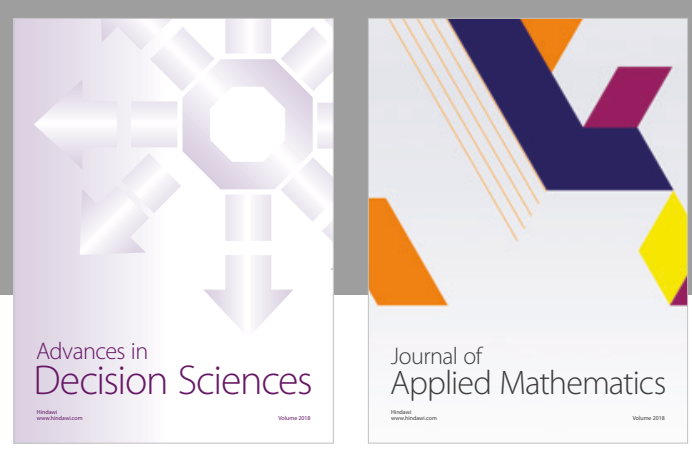

Journal of

Applied Mathematics
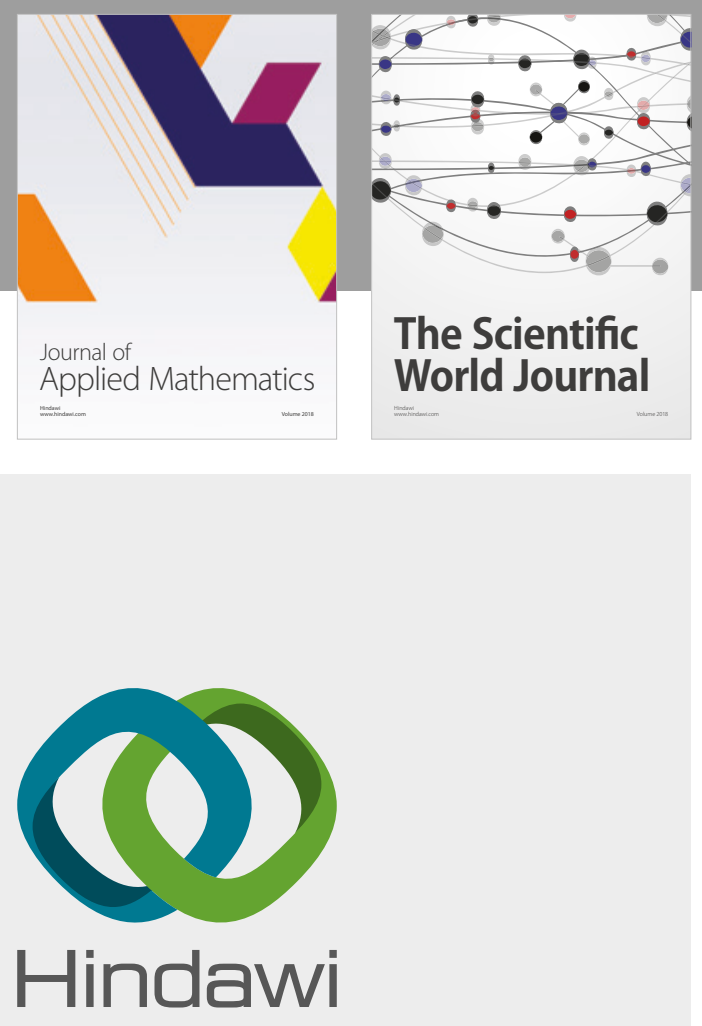

Submit your manuscripts at

www.hindawi.com

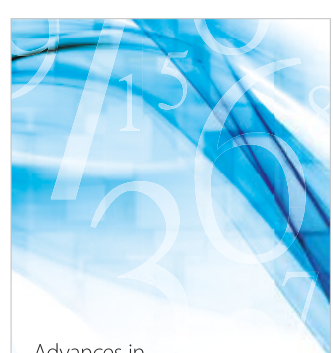

Advances in
Numerical Analysis
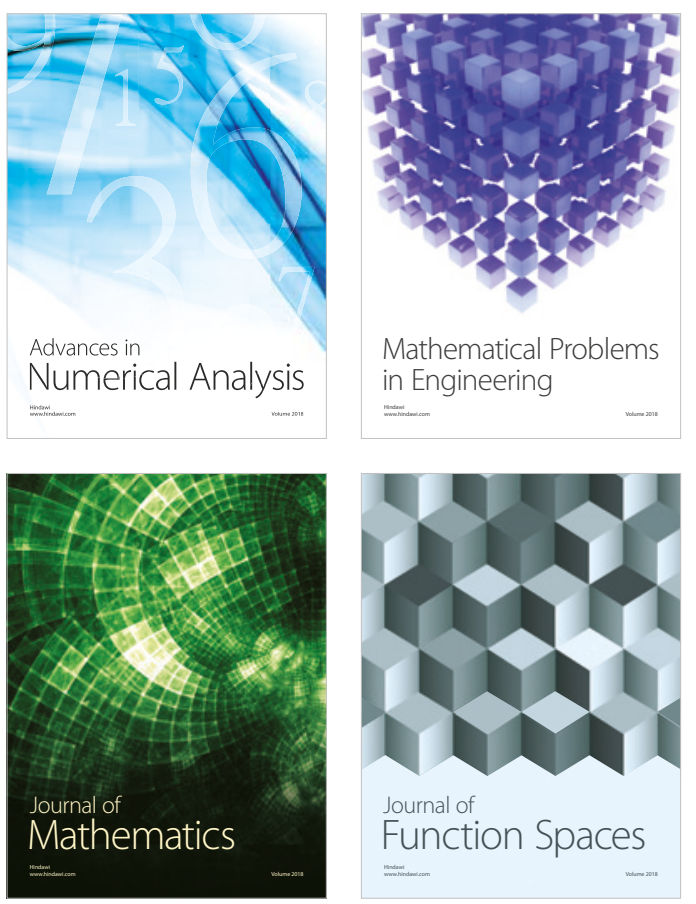

Mathematical Problems in Engineering

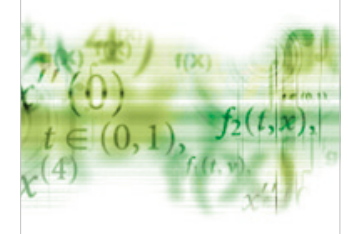

International Journal of

Differential Equations

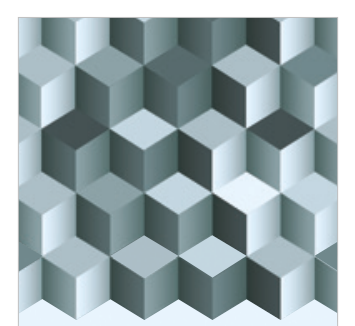

Journal of

Function Spaces

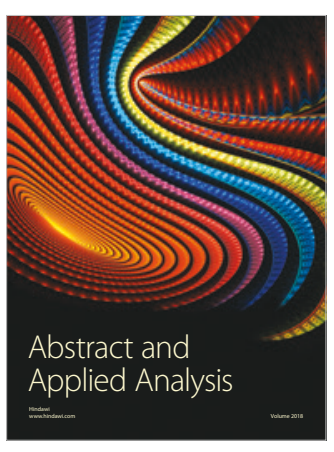

The Scientific

World Journal

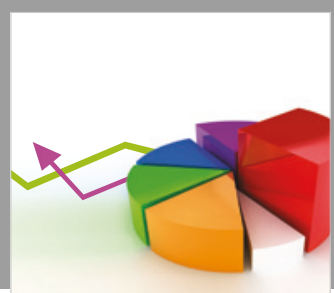

Journal of

Probability and Statistics
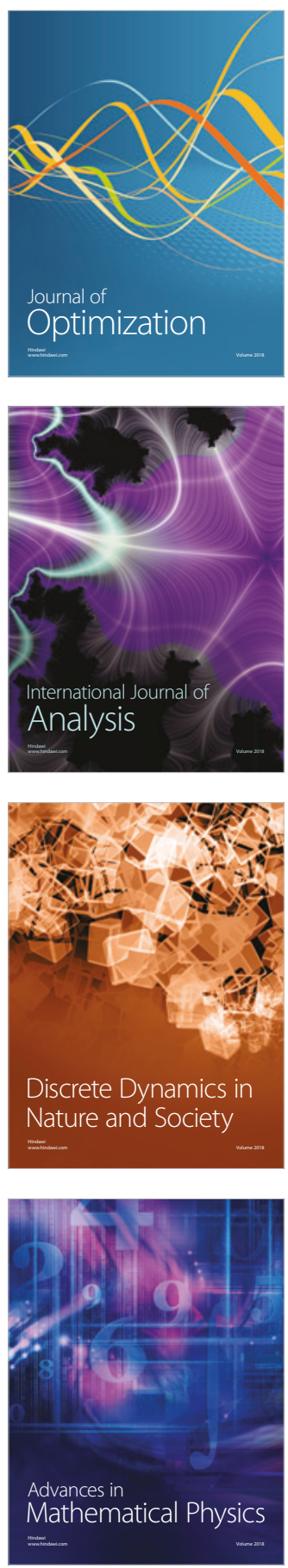